\title{
Quebec's end-of-life bill makes palliative care more transparent
}

$\mathrm{P}$ roposed legislation in Quebec to allow "medical aid in dying" to incurably ill and suffering patients contains measures that would increase oversight of palliative care in the province, say medical experts.

While a plan to allow doctors to administer life-ending medication is the most high-profile element of Bill 52, An Act Respecting End of Life Care, it also proposes a new provincial commission to oversee "all matters relating to end-of-life care." The commission would impose new requirements on hospitals and other facilities to report formally on their practices.

As it stands, hospitals in Quebec and the rest of Canada often offer palliative sedation to ease suffering. In extreme cases, doctors use "terminal sedation," in which patients are medicated into unconsciousness and deprived of artificial nutrition to expedite imminent death.

There are no uniform rules for palliative terminal sedation, says Jocelyn Downie, a professor who specializes in health, law and ethics at Dalhousie University, Halifax, Nova Scotia. Jurisdictions, hospitals and doctors often determine their own practices, she says.

This "black hole ... is a grave concern. We just don't know how much it is happening," Downie says. "It might well be happening in circumstances in which many people think it would be inappropriate or indefensible."

Dr. Marcel Boisvert, a retired palliative care physician, says he was involved in terminal sedation at least six times at Montréal's Royal Victoria Hospital. No clear rules governed the process, he says, which was handled on a case-bycase basis. Deciding whether terminal sedation is the best option is often a judgment call made by doctors, after consulting with patients and families.

Boisvert believes euthanasia is more humane because patients can live for weeks while terminally sedated, which can be agonizing for family members. "This is terrible for the family," he says. "They don't want to leave the room even to go to the bathroom."

Quebec's proposed legislation would make palliative-care treatment more transparent, say Boisvert and Downie, who strongly support the bill.

The legislation may actually lead to improved palliative care, as has happened in other jurisdictions that permit euthanasia and/or doctor-assisted suicide, says Downie. "When you bring these practices out into the open ... you have full conversations about the full spectrum of end-of-life care and [how to] do pain management better," she says.

But Dr. Gerald van Gurp, who provides palliative home care in Montréal,

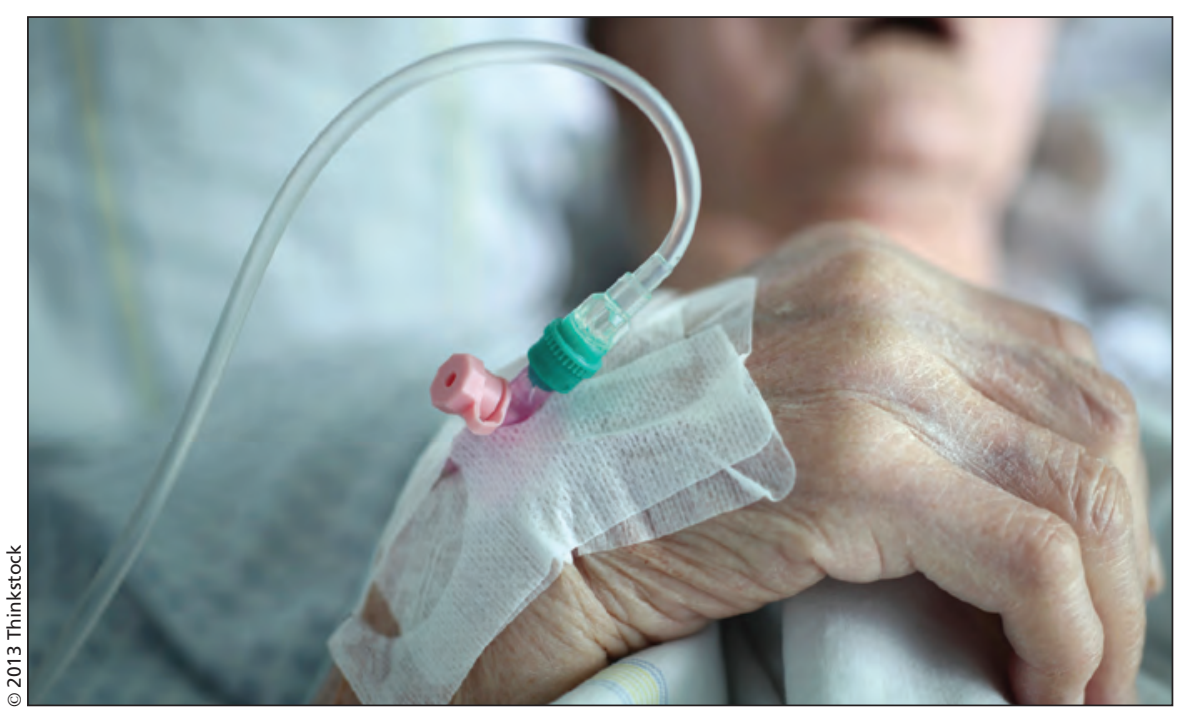

Under Bill 52, the patient would have to consent in writing to terminal sedation and two doctors would have to agree that a patient's condition qualifies for life-ending medication. 
contends the opposite is true. "In the jurisdictions where euthanasia and physician-assisted suicide have become legal, there has been less of an incentive to develop high quality palliative care. It's kind of an inverse relationship."

"The more physicians know about palliative care, the less interested they are in euthanasia," he says, adding that improving publicly funded home care to ease dying patients' suffering would negate the need for euthanasia. In his three decades of providing palliative care, he says he has never encountered a patient whose pain could not be relieved by sedatives and around-the-clock care.

Quebec's foray into medically assisted death began in 2009, when the National Assembly struck a commission to study the practice. Its 24 recommendations called for a comprehensive "dying with dignity" law. Following public hearings in 2010 and 2011, a second report was commissioned from legal experts. That report, released in January 2013, elaborated on the commission's recommendations and called on the government to create clear legislation on both assisted suicide and euthanasia

It cited a 2012 Supreme Court of British Columbia ruling that examined practices in jurisdictions that have legalized assisted suicide. It rejected the argument that assisted suicide erodes palliative care or preys on vulnerable patients who fear they may be pressured into agreeing to be injected with lethal substances. The ruling, which found an absolute ban on assisted suicide unconstitutional, is under appeal.

Four American states - Oregon, Washington, Montana and Vermont permit assisted suicide, allowing doctors to prescribe life-ending medication under strict conditions. In Europe, the Netherlands, Belgium and Luxembourg have legalized both assisted suicide and euthanasia, in which doctors administer the medication. Switzerland allows assisted suicide, but bans euthanasia.

Quebec's proposal does not permit doctor-assisted suicide, meaning that physicians can provide the life-ending medication to patients who meet the stringent criteria, but the patients must self-administer the medication.

If passed, the Quebec law permitting medically assisted death will be the first in Canada. It means that patients suffering from "constant and unbearable physical and psychological pain" from an "incurable serious illness" that is "at an advanced stage of irreversible decline" will be allowed to decide when to die. They would have to consent in writing to terminal sedation and two doctors would have to agree that a patient's condition qualifies for life-ending medication. Doctors and hospitals would have to track the administration of that sedation.

Physicians could refuse to carry out a patient's request but would be obliged to inform a supervisor so another doctor could step in. The Quebec College of Physicians backs the bill and is helping the government devise a euthanasia protocol. But a group of about 500 of Quebec's 21000 physicians sees no reason to change the legal status quo.

"I don't think the solution is to kill people; the solution is to improve endof-life care," says Dr. Catherine Ferrier, spokesperson for Total Refusal of Euthanasia in Montréal. She says she is particularly concerned about "the risk of vulnerable people being euthanized who didn't ask for it." As a doctor for geriatric patients, Ferrier says she sees a lot of elder abuse in which patients are maltreated by relatives. Also, the proposed legislation is worded so broadly that it could apply to almost anyone, she says. She is concerned that people with psychiatric illnesses and dementia are not specifically excluded.

Véronique Hivon, Quebec minister for social services and youth protection, has said she wants to start public hearings on the specifics of the bill this fall. — Janice Tibbetts, Ottawa, Ont.

CMAJ 2013. DOI:10.1503/cmaj.109-4539

\section{More News online}

Federal Wi-Fi panel criticized for undisclosed conflict: The Royal Society of Canada will reconsider its decision to appoint a University of Ottawa professor to chair a panel it convened to assess the safety of radiowave-emitting devices such as cellular phones, following a CMAJ investigation that reveals a potential conflict of interest. - Paul Christopher Webster, Toronto, Ont.

New medical marijuana regulations shift onus to doctors to prescribe: Health Canada's new medical marijuana regulations shift the onus from federal regulators to doctors and nurse practitioners to write "medical documents" similar to prescriptions authorizing patients to obtain marijuana from a federally licensed provider. — Laura Eggertson, Ottawa, Ont.

Canada's chief doctor cared for the health of a nation, but neglected his own: If David Butler-Jones could change anything about his nine years as the federal government's face of public health in Canada, he says he would have paid more attention to his own health. — Janice Tibbetts, Ottawa, Ont.

New legislation ignores health benefits of safe injection sites: A leading infection-control expert is urging public health officials to speak out about the benefits of safe injection sites instead of staying silent about new federal legislation that will make it harder for communities across Canada to establish new locations. - Laura Eggertson, Ottawa, Ont.

International alliance to share genomic data: The nascent Global Alliance to Enable Responsible Sharing of Genomic and Clinical Data is tapping into recent technological advances that have made it theoretically possible to conduct massive-scale, international genetic research. - Wendy Glausner, Toronto, Ont.

CMAJ 2013. DOI:10.1503/cmaj.109-4560 